\title{
Propuesta para aumentar los puntos experimentales en diseños D-óptimos bayesianos
}

\author{
Proposal to increase experimental points in Bayesian \\ D-optimal design
}

Cristian Fernando Téllez Piñereda

cftellezp@unal.edu.co
Víctor Ignacio López Río@

vilopez@unal.edu.co

\section{Resumen}

Uno de los criterios de uso más frecuente para la obtención de diseños óptimos es el D-optimalidad, el cual proporciona los puntos experimentales donde se minimiza el volumen del elipsoide de confianza asociado al vector de parámetros en el modelo propuesto. A diferencia del diseño D-óptimo clásico, el diseño D-óptimo bayesiano no necesariamente tiene tantos puntos de soporte como parámetros tiene el modelo. En este artículo se considera el caso en donde el diseño D-óptimo promediado por una a priori particular tiene tantos puntos de soporte como el número de parámetros del modelo. Esta situación puede no ser tan favorable cuando el modelo no se tiene especificado con total certeza, dado que no sería posible realizar pruebas de falta de ajuste para el modelo. En este artículo se propone una metodología que permite aumentar el número de puntos de soporte del diseño con el fin de que, con el diseño resultante, se pueda aplicar la prueba de bondad de ajuste. Finalmente, se ejemplifica la metodología con un modelo exponencial.

Palabras clave: D-optimalidad bayesiano, bondad de ajuste, D-eficiencia, incremento puntos experimentales, diseños óptimos.

\begin{abstract}
One of the most frequent used criteria to obtain optimal designs is D-optimality designs, which provides experimental points where the volume of confidence ellipsoid associated to the vector of parameters in the proposed model is minimized. Unlike the classical D-optimal design, the Bayesian D-optimal design does not necessarily have as many support points as the model parameters. This article considers the case where D-optimal design averaged by a specific a priori has as many support points as the number of parameters of the model. This situation
\end{abstract}

\footnotetext{
${ }^{a}$ Docente tiempo completo. Fundación Universitaria Los Libertadores, Colombia.

${ }^{\mathrm{b}}$ Profesor Asociado. Universidad Nacional de Colombia, sede Medellín, Colombia.
} 
may not be as favorable when the model is not specified with complete certainty, since it would not be possible to conduct tests due to lack of fitness for the model. This article proposes a methodology that allows increasing the number of support points of the design in order that, with the resulting design, goodness of fitness test can be applied. Finally, the methodology is exemplified with an exponential model.

Keywords: Bayesian D-optimality, goodness of fit, D-efficiency, increase in experimental points, optimal designs.

\section{Introducción}

En trabajos de investigación, usualmente se tiene el interés de modelar el comportamiento de una variable de interés $Y$ a través de un conjunto de $k$-variables explicativas $\mathbf{x}=\left(x_{1}, x_{2}, \ldots, x_{k}\right)$, por medio de un modelo estadístico que describa esta posible relación. Este modelo puede tener la forma:

$$
Y(\mathbf{x})=\eta(\mathbf{x} ; \theta)+\epsilon
$$

donde $\eta(\mathbf{x} ; \theta)$ es una función del vector de parámetros desconocido $\theta \in \mathbb{R}^{p}, x$ toma valores en un espacio de diseño $\chi$ y $\epsilon$, el error aleatorio, se asume que tiene media cero y varianza constante $\sigma^{2}$.

En este artículo se analizará un modelo no lineal, en donde la matriz de información depende de $\theta$.

La teoría de los diseños óptimos en el contexto de la estadística bayesiana presenta algunos resultados o criterios de optimalidad, que son útiles para determinar las condiciones experimentales o niveles de las covariables donde se debe experimentar para obtener estimaciones óptimas de los parámetros del modelo bajo estudio. Por ejemplo, Argumedo-Galvan \& López (2011) realizan una generalización de la metodología propuesta por O’Brien (1995) para el aumento del número de puntos de soporte en un diseño D-óptimo local. Cardona et al. (2012) realizaron una caracterización de los diseños óptimos obtenidos a través de dos funciones de utilidad asociados al D-optimalidad bayesiano para estimar en forma óptima los parámetros de dos modelos no lineales, entre otras.

Este artículo propone e implementa una estrategia que busca el aumento del número de puntos experimentales del diseño D-óptimo obtenido a partir de una distribución apriori. Para ello se adapta la propuesta de O’Brien (1995) y de Cardona et al. (2012). Se deduce explícitamente la expresión para la función de sensibilidad asociada al criterio en términos de una constante de ponderación $(\delta)$, la eficiencia que se quiere alcanzar con el nuevo diseño y el número de parámetros del modelo. Se determina la mejor elección para $\delta$, al maximizar la potencia de la prueba de falta de ajuste del modelo en estudio. Finalmente, se evaluá la bondad de la metodología en el modelo exponencial, vía simulación. 


\section{Diseño óptimo bayesiano}

A continuación se ilustran algunos conceptos básicos de la teoría clásica de diseños óptimos para modelos no lineales, y se dan resultados importantes del enfoque bayesiano asociados con algunos criterios de optimalidad.

\subsection{Diseño exacto}

Sean $x_{1}, \ldots, x_{d}$ una sucesión de $d$ puntos distintos, y $r_{i}$ el número de repeticiones del punto $x_{i}$, entonces se define un diseño exacto como una medida de probabilidad discreta $\xi_{N}$, en el espacio de diseño $\chi$, representado por:

$$
\xi=\left[\begin{array}{ccc}
x_{1} & \cdots & x_{d} \\
\frac{r_{1}}{N} & \cdots & \frac{r_{d}}{N}
\end{array}\right],
$$

donde $\sum_{i=1}^{d} r_{i}=N$, siendo $N$ el número total de corridas experimentales. La primera fila denota los puntos del espacio de diseño, $\chi$, donde toman las mediciones de la variable respuesta y la segunda fila contiene los pesos $w_{i}=\xi\left(x_{i}\right)=\frac{r_{i}}{N}$, indicando la proporción de mediciones para ser tomadas en cada punto.

\subsection{Diseño continuo}

A diferencia del diseño exacto, los pesos $w_{i}$ pueden ser cualquier número entre cero y uno, no se exige que sean números racionales. Aunque en la práctica todos los diseños son exactos, desde el punto de vista de la obtención de estos es recomendable usar los diseños aproximados por las ventajas que ofrece el usar el análisis convexo.

\subsection{Matriz de información}

Para cada diseño $\xi$ se define la matriz de información:

$$
M(\xi ; \theta)=\int_{\chi} f(x ; \theta) f^{T}(x ; \theta) d \xi(x),
$$

y como el diseño es una medida discreta con soporte finito, entonces (3) es equivalente a:

$$
M(\xi ; \theta)=\sum_{i=1}^{d} f\left(x_{i} ; \theta\right) f^{T}\left(x_{i} ; \theta\right) w_{i}
$$

donde $f(x ; \theta)=\frac{\partial \eta(x ; \theta)}{\partial \theta}$. En este caso la matriz de información depende del vector de parámetros, $\theta$. 


\subsection{Criterios de optimalidad}

La teoría de diseños óptimos, Kiefer (1959), está relacionada con la selección de un diseño $\xi$ que maximiza algún funcional de la matriz de información. Así, los criterios de optimalidad (López \& Ramos 2007) son definidos como juicios que maximizan algún funcional real (con un significado estadístico) de la matriz de información sobre la clase de todos los diseños aproximados definidos en $\chi$.

- El criterio D-optimalidad se define como el siguiente funcional:

$$
\begin{aligned}
\psi(\xi) & :=\psi(M(\xi)) \\
& =\left|M^{-1}(\xi ; \theta)\right| .
\end{aligned}
$$

Busca aquel diseño que minimiza un funcional escalar de la varianza generalizada asintótica asociada a los estimadores de máxima verosimilitud del vector de parámetros dada por:

$$
\operatorname{det}\left(M^{-1}(\xi)\right),
$$

donde $\operatorname{det}(A)$ denota la función determinante de la matriz A. Según lo expuesto en Atkinson et al. (2007), es más conveniente el uso de una función convexa dada por el logaritmo natural, puesto que se facilitan los cálculos y además la función logaritmo natural del determinante es convexa, así un diseño $\xi_{D}$ se dice que es D-óptimo si minimiza - $\log (|M(\xi)|)$ o equivalentemente maximiza $\log (|M(\xi)|)$.

- El criterio G-optimalidad, propuesto por Kiefer (1959), consiste en encontrar el diseño que minimiza la varianza de la respuesta predicha más grande. Es decir, un diseño $\xi^{*}$ es $G$-óptimo si minimiza el máximo de la función $d(x ; \xi, \theta)$ sobre todo los $x \in \chi$. Es decir,

$$
\min _{\xi} \operatorname{máx}_{x \in \chi} d(x ; \xi, \theta)=\operatorname{máx}_{x \in \chi} d\left(x ; \xi^{*}, \theta\right)
$$

donde $d(x ; \xi, \theta)=f^{T}(x ; \theta) M^{-1}(\xi ; \theta) f(x ; \theta)$ representa la función de varianza de la respuesta predicha estandarizada.

- Existen otros criterios de optimalidad que no se exploran en este artículo, para ello se pueden consultar los trabajos de López \& Ramos (2007), Atkinson et al. (2007).

\subsection{Teorema de equivalencia para un diseño D-óptimo}

Kiefer \& Wolfowitz (1959) mostraron la equivalencia entre el criterio D-optimalidad y G-optimalidad, el cual se puede enunciar de la siguiente manera:

Un diseño $\xi^{*}$ con matriz de momentos $M\left(\xi^{*}\right)$, definida positiva, es D-óptimo sí y solo si es G-óptimo sí y solo si: 


$$
\operatorname{máx}_{x \in \chi} d\left(x ; \xi^{*}\right)=p .
$$

Donde $p$ es el número de parámetros del modelo.

El máximo de la función de varianza de la respuesta predicha se obtiene en los puntos de soporte del diseño D-óptimo y es igual al número de parámetros del modelo, en caso de D-optimalidad, el peso de cada punto de soporte es inversamente proporcional a $p, \omega_{i}=\frac{1}{p}$.

\subsection{Eficiencia de un diseño}

Una forma de medir la eficiencia de un diseño, $\xi$, con respecto al diseño $D_{\pi}(\xi)$ óptimo, $\xi^{*}$, es a partir del cociente del criterio de optimalidad evaluado tanto en $\xi$, como $\xi^{*}$, es decir,

$$
e f_{D_{\pi}}(\xi)=\frac{D_{\pi}(\xi)}{D_{\pi}\left(\xi^{*}\right)}
$$

de donde $0<e f_{D_{\pi}}(\xi) \leq 1$. Un diseño $\xi$ es comparable con un diseño $D_{\pi}$-óptimo en la medida que su eficiencia sea cercana a uno, (ver López (2008)).

$$
e f_{D}(\xi)=\left[\frac{|M(\xi ; \theta)|}{\left|M\left(\xi^{*} ; \theta\right)\right|}\right]^{1 / p}
$$

Al tomar la $p$-ésima raíz cuadrada se obtiene una medida de eficiencia que tiene las dimensiones de una razón de varianza. La $e f_{D}(\xi)$ permite determinar la capacidad del diseño $\xi$ para estimar de manera eficiente los parámetros del modelo en comparación con el diseño D-óptimo, $\xi_{D}^{*}$. La D-eficiencia se puede interpretar como el número de réplicas requeridas del diseño para que sea tan eficiente como el diseño D-óptimo (Atkinson et al. 2007).

\subsection{Criterio D-optimalidad bayesiano}

Los diseños D-óptimos locales maximizan $\log \left|M\left(\xi ; \theta_{0}\right)\right|$, donde $\theta_{0}$ representa un valor apriori de $\theta$. Si además es posible tener una distribución apriori $\pi(\theta)$ para el vector de parámetros $\theta$, el diseño D-óptimo promediado $\pi(\theta)$ puede ser obtenido al maximizar:

$$
D_{\pi}(\xi)=\int_{\Theta} \log |M(\xi ; \theta)| \pi(\theta) d \theta
$$


donde $\Theta$ es el soporte de la distribución $\pi(\theta)$. Para este criterio, se define la función de sensibilidad para un diseño $\xi, d(x ; \xi)$, como:

$$
d(x ; \xi)=\int_{\Theta} d(x ; \xi, \theta) \pi(\theta) d \theta
$$

donde $d(x ; \xi, \theta)=f^{T}(x ; \theta) M^{-1}(\xi ; \theta) f(x ; \theta)$. Un diseño que maximice la función dada en (9) se denomina diseño $\Psi_{\pi}$-óptimo.

La matriz de información para un diseño D-óptimo bayesiano, la cual depende de un vector de parámetros $\theta$. está dada por:

$$
\begin{aligned}
M(\xi ; \theta) & =\int_{\chi} f(x ; \theta) f^{T}(x ; \theta) \xi(d x), \\
& ==\sum_{x \in \operatorname{Soporte}(\xi)} f(x ; \theta) f^{T}(x ; \theta) \xi(x),
\end{aligned}
$$

Para este caso el teorema de equivalencia asociado es: un diseño $\xi^{*}$ es $D_{\pi^{-}}$óptimo si $\phi(x ; \xi)=p-\int_{\Theta} \operatorname{tr}\left[f^{T}(x ; \theta) M^{-1}\left(\xi^{*}: \theta\right) f(x ; \theta)\right] \pi(\theta) \leq 0$ y la igualdad se cumple en los puntos de soporte del diseño.

La función $\phi(x ; \xi)$ se conoce como la derivada direccional asociada al criterio de optimalidad y varía dependiendo del criterio utilizado (Atkinson et al. 2007).

En la Tabla 1 se muestra la derivada direccional asociada a diferentes versiones del criterio de optimalidad bayesiano.

Tabla 1: Versiones del criterio de optimalidad bayesianos junto con su respectiva derivada direccional asociada. Fuente: elaboración propia.

\begin{tabular}{ccc}
\hline Criterio & $\Psi\{M(\xi ; \theta)\}$ & Derivada direccional $\phi(x ; \xi)$ \\
\hline I & $E_{\theta} \log \left[\left|M^{-1}\right|\right]$ & $p-E_{\theta}\left\{\operatorname{tr} M^{-1} M\left(\xi_{x} ; \theta\right)\right\}$ \\
II & $\log \left[E_{\theta}\left(\left|M^{-1}\right|\right)\right]$ & $p-E_{\theta}\left\{\left|M^{-1}\right| \operatorname{tr} M^{-1} M\left(\xi_{x} ; \theta\right) / E_{\theta}\left|M^{-1}\right|\right\}$ \\
III & $\log \left[\left|E_{\theta}\left(M^{-1}\right)\right|\right]$ & $p-E_{\theta}\left\{\operatorname{tr} M^{-1} E_{\theta}\left(M^{-1}\right) M^{-1} M\left(\xi_{x} ; \theta\right)\right\}$ \\
IV & $\log \left[E_{\theta}(|M|)\right]^{-1}$ & $p-E_{\theta}\left\{|M| \operatorname{tr} M^{-1} M(\bar{\xi} ; \theta) / E_{\theta}|M|\right\}$ \\
V & $\log \left[\left|E_{\theta}(M)\right|\right]^{-1}$ & $p-\operatorname{tr}\left(E_{\theta}(M)\right)^{-1} M\left(\xi_{x} ; \theta\right)$ \\
\hline
\end{tabular}

donde $M^{-1}=M^{-1}(\xi ; \theta)$. En este artículo se utiliza la versión $I$.

\section{El problema de la falta de ajuste en el diseño D-óptimo}

La estrategia para obtener diseños con puntos extras ha sido trabajada por O'Brien (1992), O'Brien (1995), Chaloner \& Larntz (1989), entre otros. O'Brien (1995) 
recomienda la siguiente estrategia para obtener diseños con puntos de soporte extras:

\subsection{Algoritmo}

1. Encontrar el diseño D-óptimo local, $\xi_{D}$. Este diseño tendrá $p$ puntos de soporte (Gaffke 1987).

2. Construir el diseño:

$$
\xi_{N}=\frac{p}{p+1} \xi_{D}+\frac{1}{p+1} \xi_{x},
$$

donde $\xi_{D}$ es el diseño D-óptimo local encontrado (1) y $\xi_{x}$ es un diseño con un solo punto, es decir, tiene concentrado todo su peso en el punto $x$.

3. Fijar un valor mínimo para la eficiencia que se desea alcanzar con el nuevo diseño, denotado por de, valor entre 0 y 1 , y encontrar los $t$ valores de $x$ tal que:

$$
d\left(x ; \xi_{D}, \theta^{0}\right)=p\left[\left(\frac{p+1}{p} d e\right)^{p}-1\right] .
$$

4. Por último, tomar como diseño seudo-óptimo aquel diseño que tenga $r_{1}$ réplicas en los $p$ puntos de soporte del diseño D-óptimo $\xi_{D}, \mathrm{y} r_{2}$ réplicas en los $t$ puntos de soporte obtenidos en el paso anterior.

La propuesta de O'Brien (1995) da igual peso a cada punto de soporte del nuevo diseño, $\xi_{N}$, con lo cual surgen los siguientes interrogantes: ¿Qué ocurre si la ponderación dada a los nuevos puntos es $\delta$ y a los puntos del diseño $\Psi_{\pi}$-óptimo es $1-\delta$ ? ¿Qué estrategias se pueden proponer para la escogencia del valor de $\delta$ ?

La respuesta a ambos interrogantes será el objetivo de las siguientes secciones.

\section{Generalización}

Se propone construir el nuevo diseño a partir de:

$$
\xi_{N}=(1-\delta) \xi_{D}+\delta \xi_{x}
$$

o de forma explícita

$$
\xi_{N}=\left\{\begin{array}{cccc}
x_{1} & \cdots & x_{p} & x \\
\frac{1-\delta}{p} & \cdots & \frac{1-\delta}{p} & \delta
\end{array}\right\}
$$


Donde $\xi_{D}$ es el diseño $D_{\pi}$-óptimo asumiendo que este tiene $p$ puntos de soporte, la constante $\delta$ determina el peso en el diseño $\xi_{x}$, diseño que tiene su peso concentrado en el punto $x$.

Una expresión equivalente para la función de sensibilidad del diseño $D_{\pi}$ usando el nuevo diseño $\xi_{N}=(1-\delta) \xi_{D}+\delta \xi_{x}$ y asumiendo la D-eficiencia del diseño $\xi_{N}$ fija se puede escribir como:

$$
d\left(x ; \xi_{D}\right)=\int\left(\left|M\left(\xi_{D} ; \theta\right)\right|^{m}-1\right) \frac{1-\delta}{\delta} \pi(\theta) d \theta .
$$

con $m=\varepsilon_{f_{\xi_{D}}}^{p}\left(\xi_{N}\right)-1, \varepsilon_{f_{\xi_{D}}}\left(\xi_{N}\right)$ la $D_{\pi}$ eficiencia del diseño $\xi_{N}$ y $p$ el número de parámetros del modelo.

Con los $x$ 's que satisfacen la ecuación (16) y los puntos de soporte del diseño $D_{\pi^{-}}$ óptimo, se forma el nuevo diseño $\xi_{N}$ el cual tendrá los $p$ puntos del diseño $D_{\pi} \mathrm{y}$ los $s$ nuevos puntos adicionales, es decir, tendrá $p+s$ puntos de soporte.

A continuación se muestra un algoritmo para la escogencia del $\delta$.

\subsection{Escogencia del $\delta$}

A partir del diseño $D_{\pi}$-óptimo, el número de corridas experimentales $(N)$, la eficiencia del diseño $\xi_{N}$ y una rejilla fija para $\delta$, se encuentra el diseño $\xi_{i N}$ dado por $\left(1-\delta_{i}\right) \xi_{D}+\delta_{i} \xi_{x}$ para un $\delta_{i}$ fijo en la rejilla. El diseño $\xi_{N}$ es transformado a un diseño exacto de tamaño $N$ mediante la metodología de Fedorov \& Hackl (1997).

Dado los diseños exactos asociados a cada $\delta$ en la rejilla, se define una estrategia para determinar el peso $\delta$ (lo cual implica escoger uno o varios diseños) de tal forma que el diseño resultante maximice la potencia de la prueba de falta de ajuste del modelo propuesto. El algoritmo para calcular la potencia de la prueba de falta de ajuste se desarrolla en dos pasos. En el primer paso se encuentra una distribución empírica para el estadístico $-2 \log (\Lambda)$ para muestras pequeñas, donde $\Lambda$ es la razón de verosimilitud entre el modelo que se asume es correcto (modelo bajo $H_{0}$ ) y un modelo distinto al considerado, en el cuál el conjunto de parámetros sea subconjunto del modelo anterior (modelo bajo $H_{1}$ ). En el segundo paso se calcula la potencia de la prueba. Es decir,

\section{Distribución del estadístico $-2 \log (\Lambda)$ en muestras pequeñas}

1. Fijar un número de simulaciones $N_{\text {sim }}$.

2. Para el diseño exacto se calculan las medias; estas se calculan evaluando los puntos de soporte del diseño exacto y usando como estimación de $\theta$ su valor local, es decir,

$$
\mu_{i}=\eta\left(x_{i}, \theta_{0}\right),
$$

donde $x_{i}$ son los puntos de soporte del diseño. 
3. Para el diseño exacto, se simulan observaciones de la variable respuesta según el modelo para trabajar (Modelo bajo $H_{0}$ cierta en la prueba de falta de ajuste), para el que se asume que el término del error es normal con media cero, varianza constante e independientes y tomando como varianza para el error la obtenida en un estudio previo.

4. Para las observaciones de la variable respuesta generada en el paso 3 y los puntos de soporte del diseño exacto, se hace el ajuste del modelo propuesto mediante mínimos cuadrados no lineales (ajuste bajo $H_{0}$ cierta).

5. Para el ajuste en el paso 4, se calcula el estadístico:

$$
\chi_{o}=-2 \log (\Lambda)
$$

donde $\Lambda$ es la razón de verosimilitudes entre el modelo bajo $H_{0}$ (modelo propuesto) y el modelo bajo $H_{1}$ (modelo general) en la prueba de falta de ajuste.

6. Para el número de simulaciones fijo $N_{\text {sim }}$ se computa un vector de cuantiles al 1, 5 y $10 \%$ de la distribución empírica $-2 \log (\Lambda)\left(Q_{e m p A}\right)$, luego se incrementa ese número de simulaciones, por ejemplo el doble y se computa otro vector de cuantiles al 1,5 y $10 \%\left(Q_{e m p B}\right)$ y fijando un error se calcula la norma euclidiana:

$$
\left\|Q_{e m p A}-Q_{e m p B}\right\|<\epsilon,
$$

Si la diferencia en la ecuación (19) es pequeña, el número de simulaciones $N_{\text {sim }}$ para la distribución empírica de $-2 \log (\Lambda)$ es aquella con la que se obtuvo $Q_{e m p A}$, de lo contrario se incrementa y así sucesivamente hasta que se cumpla la ecuación (19).

7. Se repiten los pasos 5 y 6 un número $N_{\text {sim }}$ de veces y se encuentra la distribución empírica del estadístico $-2 \log (\Lambda)$ para muestras pequeñas.

\section{Cálculo de la potencia de la prueba de falta de ajuste del modelo}

1. Se perturban las medias obtenidas en el paso 2 de la estimación de la distribución empírica con un $\gamma$ fijo; siendo $\gamma$ el factor de perturbación de las medias; adicionalmente, se toma el cuadrado medio del error (MSE) obtenido en un estudio previo como varianza para el error. Para el diseño exacto, se simulan observaciones de la variable respuesta conforme el modelo para trabajar, donde se asume que el término del error es normal con media cero, varianza constante y además independiente (modelo bajo $H_{1}$ cierto), es decir,

$$
Y_{i p}=\mu_{i p}+\epsilon_{i}
$$

donde $\mu_{i p}=\mu_{i} \pm \gamma_{j}$, con $\gamma_{j}$ una perturbación fija. Las medias perturbadas para el diseño exacto, $\epsilon_{i} \sim N(0, M S E)$ y $Y_{i p}$ las respuestas simuladas bajo $H_{1}$ para el diseño. 
2. Para el diseño exacto, con cada perturbación de las medias, se hace el ajuste del modelo propuesto mediante mínimos cuadrados no lineales (ajuste bajo $H_{0}$ falsa), es decir, se tienen tantos ajustes como perturbaciones hayan considerado.

3. Para cada ajuste en el paso 2 , se calcula el estadístico $\chi_{0}$ dado en la ecuación (18).

4. Se repiten los pasos del 1 al $3 N_{\text {sim }}$ veces y a partir de esto se obtiene la potencia de la prueba de falta de ajuste para los niveles de significancia del 1, 5 y $10 \%$ para el diseño exacto mediante:

$$
\begin{aligned}
\text { Potencia de la prueba } & =P\left(\text { rechazar } H_{0} \mid H_{0} \text { falsa }\right) \\
& =P\left(\chi_{0}>Q_{E m p i}\right) \\
& =\frac{\# \text { de rechazos de la prueba }}{N_{\text {sim }}}
\end{aligned}
$$

Donde $Q_{E m p i}$ es el vector de cuantiles calculado de la distribución empírica al 1, 5 y $10 \%$. Se tendrá por cada nivel de significancia tantas potencias como perturbaciones haya.

5. Se promedian las potencias obtenidas en el paso 2 por cada nivel de significancia y se toman estas como valor representativo.

- Los procedimientos $I$ y $I I$ deben ser repetidos para cada uno de los $\delta$ 's de la rejilla.

\section{Aplicación de la metodología}

Como una aplicación para evidenciar los resultados obtenidos en este artículo, se utiliza un modelo de decrecimiento exponencial en un estudio que investiga la acción conjunta de mezclas de ácidos fenólicos en la inhibición del crecimiento de las raíces del tipo de hierba perennial ryegrass (Lolium perenne L.) y su significado en investigación alelopática, datos tomados de Inderjit \& Olofsdotter (2002). El término alelopática se refiere a los efectos perjudiciales o benéficos que son directa o indirectamente el resultado de la acción de compuestos químicos que, liberados por una planta, ejercen su acción en otra.

La idea es entonces, obtener diseños que maximicen la prueba de falta de ajuste para el modelo de decrecimiento exponencial. Para obtener estos diseños se tomó como distribución apriori, una distribución uniforme discreta alrededor de los valores locales $\Theta^{T}=\left[\theta_{0}, \theta_{1}\right]$, con el fin de incorporar información asociada al desconocimiento de estos parámetros. A continuación se muestra el procedimiento para encontrar dichos diseños. 


\subsection{Modelo y estimación del modelo}

Los datos correspondientes al estudio de la toxicidad del ácido ferúlico son presentados en la Figura 1, donde se observa la tendencia exponencial de la longitud de la raíz en función de la concentración.

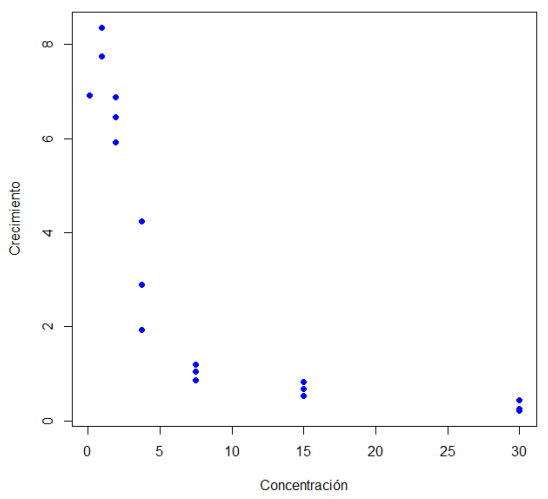

Figura 1: Dispersión de los datos de longitud de la raíz en términos de la concentración de ácido ferúlico. Fuente: elaboración propia.

La parte determinística del modelo propuesto para explicar la relación entre estas dos variables es la siguiente:

$$
\eta\left(x, \theta_{0}, \theta_{1}\right)=\theta_{0} \exp \left(-\frac{x}{\theta_{1}}\right)
$$

donde $x$ es la concentración de ácido en la planta y la respuesta del modelo es la longitud de la raíz. En el modelo anterior la función que relaciona la variable explicativa con la variable respuesta es no lineal en los parámetros, los valores ajustados de $\theta_{0}$ y $\theta_{1}$ se obtuvieron con la función nls de la librería nlstools del paquete $\mathrm{R}$, estos se muestran en la Tabla 2 y el modelo ajustado se muestra en la Figura 2,

Tabla 2: Parámetros estimados por mínimos cuadrados no lineales para el modelo de decrecimiento exponencial. Fuente: elaboración propia.

\begin{tabular}{ccccc}
\hline & Estimación & Error estándar & Valor t & $\operatorname{Pr}(>|t|)$ \\
\hline$\theta_{0}$ & 9.2 & 0.65 & 13.98 & 0.00 \\
$\theta_{1}$ & 4.1 & 0.58 & 7.03 & 0.00 \\
\hline
\end{tabular}




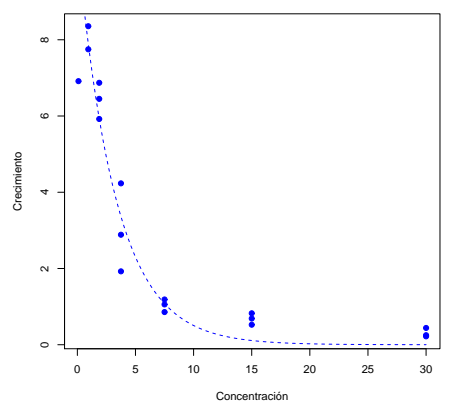

Figura 2: Curva ajustada para modelo de decrecimiento exponencial. Fuente: elaboración propia.

\subsection{Diseño $D_{\pi}$-óptimo para el modelo de decrecimiento exponencial}

A continuación se mostrará el procedimiento para hallar un diseño $D_{\pi}$-óptimo cuando la distribución $\pi$ es una distribución uniforme discreta. En este caso el diseño $D_{\pi}$-óptimo será aquel que minimice la siguiente función:

$$
\psi(\xi)=\int_{\Theta}-\log |M(\xi, \theta)| d \pi(\theta)=\sum_{i=1}^{d}-\log \left|M\left(\xi, \theta_{i}\right)\right| \frac{1}{d}
$$

donde $d$ es el número de puntos de la distribución uniforme considerada. Los datos de la distribución uniforme considerada son mostrados en la Tabla 3.

Tabla 3: Observaciones de la distribución uniforme. Fuente: elaboración propia

\begin{tabular}{cc}
\hline$\theta_{0}$ & $\theta 1$ \\
\hline 9.44667 & 3.6234 \\
10.49630 & 3.6234 \\
13.64519 & 3.6234 \\
9.44667 & 3.2940 \\
10.49630 & 3.2940 \\
13.64519 & 3.2940 \\
9.44667 & 2.3058 \\
10.49630 & 2.3058 \\
13.64519 & 2.3058 \\
\hline
\end{tabular}


Para obtener la matriz de información se requiere calcular las derivadas de la función $\eta$ con respecto a cada parámetro, dichas derivadas son mostradas en el siguiente vector:

$$
f\left(x ; \theta_{0}, \theta_{1}\right)=\left[\exp \left(-\frac{x}{\theta_{1}}\right), \frac{\theta_{0} x \exp \left(-\frac{x}{\theta_{1}}\right)}{\theta_{1}^{2}}\right]^{T} .
$$

Para minimizar la función dada en la ecuación (22), se utilizó la función nlminb de la librería DEoptim del paquete estadístico $\mathrm{R}$ Development Core Team. Se verificó que la función de sensibilidad asociada alcanzaba su máximo en $p=2$, número de parámetros del modelo y el máximo se alcanza en los puntos de soporte del diseño dado en la ecuación (24):

$$
\xi_{D}=\left\{\begin{array}{cc}
0.94 & 3.827 \\
0.5 & 0.5
\end{array}\right\}
$$

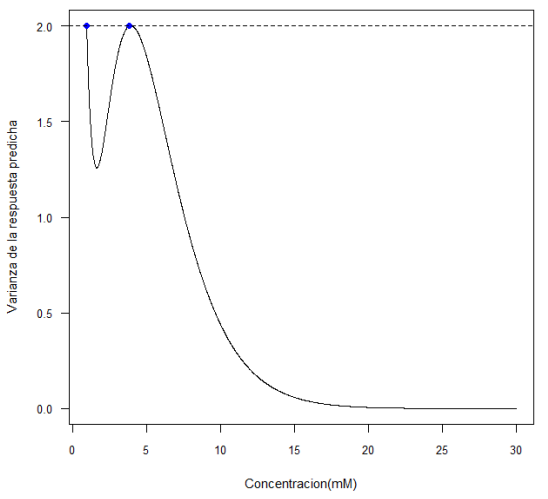

Figura 3: Gráfico de la función de sensibilidad $d\left(t, \xi_{D}, \theta_{0}\right)$ modelo de decrecimiento exponencial. Fuente: elaboración propia.

\subsection{Diseños seudoóptimos utilizando la metodología propuesta}

El diseño $D_{\pi}$-óptimo, dado en la ecuación (24) solo tiene dos puntos de soporte, con los cuales no es posible realizar pruebas de bondad de ajuste. A continuación se mostrará el procedimiento para aumentar dicho número a partir de la metodología propuesta en la sección 4.1 . 
En la Tabla 4 se muestran los diseños seudo-óptimos donde se incorpora el nuevo punto que satisface la ecuación (16) donde $\delta \in(0.285,0.5)$ con incrementos de 0.05 , $N=20$ corridas experimentales y $E f_{\xi_{D_{\pi}}}\left(\xi_{N}\right)=0.95$. Los límites del intervalo para $\delta$ se escogieron de tal forma que existiera solución para la ecuación (16) y con la condición de que el nuevo diseño otorgara al menos el $50 \%$ al diseño $D$-óptimo.

Tabla 4: Diseños seudo-óptimos con tres puntos de soporte. Fuente: elaboración propia

\begin{tabular}{cc}
\hline$\delta$ & \\
\hline 0.285 & $\xi_{N_{1}}=\left\{\begin{array}{ccc}0.94 & 3.827 & 6.822 \\
0.35 & 0.35 & 0.3\end{array}\right\}$ \\
0.335 & $\xi_{N_{2}}=\left\{\begin{array}{ccc}0.94 & 3.827 & 7.63 \\
0.35 & 0.35 & 0.3\end{array}\right\}$ \\
0.385 & $\xi_{N_{3}}=\left\{\begin{array}{ccc}0.94 & 3.827 & 8.314 \\
0.3 & 0.3 & 0.4\end{array}\right\}$ \\
0.435 & $\xi_{N_{4}}=\left\{\begin{array}{ccc}0.94 & 3.827 & 8.93 \\
0.27 & 0.27 & 0.46 \\
0.94 & 3.827 & 9.50 \\
0.25 & 0.25 & 0.5\end{array}\right\}$ \\
0.485 & $\xi_{N_{5}}=\{$
\end{tabular}

\subsection{Distribución empírica del estadístico $-2 \log (\Lambda)$ y cálculo de potencia de los diseños seudoóptimos}

La metodología consiste en escoger los diseños que maximicen la prueba de falta de ajuste del modelo. Siguiendo el algoritmo mostrado en la sección 4.1. En la Tabla 5 se muestra el número de simulaciones y la diferencia, en norma, de los cuantiles calculados con las distribuciones empíricas.

De acuerdo con los resultados de la Tabla 5 y con un $\epsilon=0.05$, el número de simulaciones apropiado para encontrar la distribución empírica del estadístico $-2 \log (\Lambda)$ es $N_{\text {sim }}=10000$ para los diseños $\xi_{N_{1}}$ hasta $\xi_{N_{4}}$ y para el diseño $\xi_{N_{5}}$ es de $N_{\text {sim }}=5000$.

Tabla 5: Normas de las diferencias de los cuantiles para distintos $N_{\text {empi }}$. Fuente: elaboración propia

\begin{tabular}{cccccc}
\hline$N_{e m p i}$ & \multicolumn{6}{c}{$\left\|Q_{e m p A,(1-\alpha)}-Q_{e m p D,(1-\alpha)}\right\|$} \\
\hline & $\xi_{N_{1}}$ & $\xi_{N_{2}}$ & $\xi_{N_{3}}$ & $\xi_{N_{4}}$ & $\xi_{N_{5}}$ \\
500 & 0.26 & 0.36 & 0.231 & 0.31 & 0.16 \\
1000 & 0.20 & 0.27 & 0.20 & 0.24 & 0.09 \\
2000 & 0.11 & 0.14 & 0.128 & 0.16 & 0.07 \\
5000 & 0.08 & 0.09 & 0.11 & 0.07 & 0.028 \\
10000 & 0.03 & 0.012 & 0.02 & 0.019 & 0.0152 \\
\hline
\end{tabular}


Para determinar la mejor elección de la constante de ponderación $\delta$, se realiza un estudio de simulación descrito en la sección 4.1, de tal forma que el diseño para escoger será aquel que maximice la potencia de la prueba de falta de ajuste.

Después de realizar el proceso de simulación propuesto se halla el gráfico de las potencias para los cinco diseños seudo-óptimos con diferentes valores de $\alpha$, ver Figura 4. Se observa que el diseño seudo-óptimo que presenta la mayor potencia de la prueba de falta de ajuste es el diseño $\xi_{N_{1}}$ el cual es:

$$
\xi_{N_{1}}=\left\{\begin{array}{ccc}
0.94 & 3.827 & 6.822 \\
0.35 & 0.35 & 0.3
\end{array}\right\}
$$

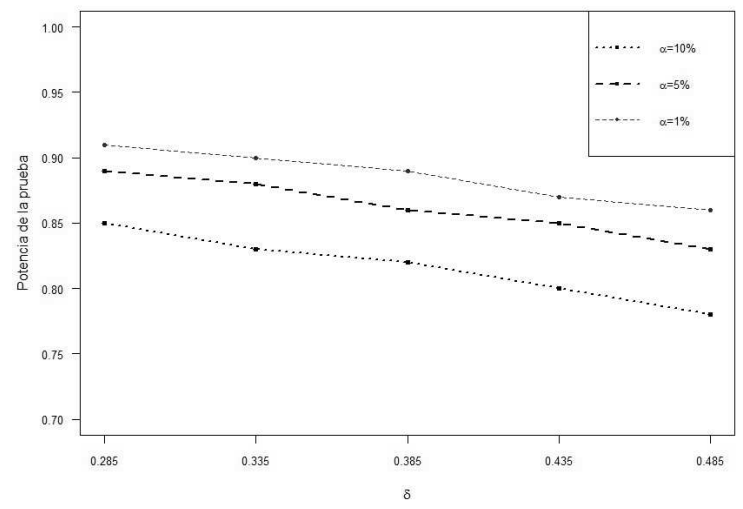

Figura 4: Potencia de la prueba de falta de ajuste con $N=20$. Fuente: elaboración propia.

Este diseño permite validar el supuesto de falta de ajuste del modelo, lo cual como ya se mencionó, es muy útil cuando no se tiene certeza del modelo. También, se interpreta de la siguiente manera: de las 20 corridas experimentales, siete se deben realizar con una concentración de $0.94 \mathrm{mM}$, otras siete con una concentración de 3.827 mM y las 6 restantes se harán con una concentración 6.822 mM.

En la Figura 5 se muestra el gráfico de dispersión con los puntos de soporte del diseño (25).

\section{Conclusión y discusión}

A partir del estudio realizado en este artículo, con el fin de aumentar el número de puntos de soporte en un diseño $D_{\pi}$-óptimo se puede concluir lo siguiente: 


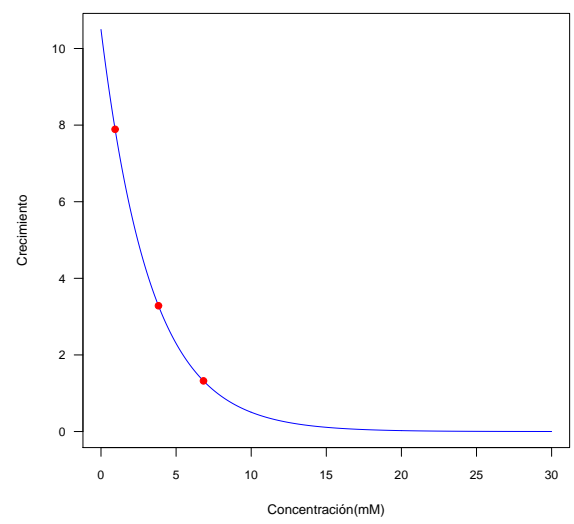

Figura 5: Puntos de soportes del diseño seudo-óptimo. Fuente: elaboración propia.

- Se realizó una generalización de la metodología de O’Brien (1995) para adicionar puntos de soporte a los diseños D-óptimos, desde el punto de vista de los diseños $D_{\pi}$-óptimo, se encontró una expresión para la función de sensibilidad en términos del número de parámetros $p$, la constante de ponderación $\delta$, y la eficiencia del diseño $\xi_{N}$. Esta expresión fue utilizada para encontrar los diseños seudo-óptimos con más de $p$ puntos de soporte, que permitan validar el supuesto de falta de ajuste del modelo.

- Para el modelo de decrecimiento exponencial se encontró que, utilizando la metodología, todos los diseños resultantes tienen potencias altas.

- Es indispensable seguir investigando con otras alternativas para el aumento del número de puntos de soporte del diseño óptimo y proponer otras metodologías para otros criterios de optimalidad, como por ejemplo, c-optimalidad, entre otros.

Recibido: 18 de abril de 2013 Aceptado: 1 de agosto de 2013

\section{Referencias}

Argumedo-Galvan, S. \& López, V. (2011), 'Estrategia de adición de puntos de soporte para un diseño D-óptimo', VIII Coloquio Internacional de Estadística Métodos Estadísticos Aplicados a Finanzas y Gestión de Riesgo pp. 1-17.

Atkinson, A., Donev, A. \& R., T. (2007), Optimum experimental designs with SAS, Oxford University Press, New York. 
Cardona, J., López, V. I. \& Correa, J. C. (2012), 'Diseños óptimos bayesianos para estimación de parámetros en farmacocinética', Comunicaciones en Estadística 5(1), 97-112.

Chaloner, K. \& Larntz (1989), 'Optimal bayesian design applied to logistic regression experiments', Journal of Statistical 1, 191-208.

Fedorov, V. \& Hackl, P. (1997), 'Model oriented design of experiments.', Lecture Notes in Statistics .

Gaffke, N. (1987), 'On D-optimality of exact linear regression designs with minimum support', Journal of Statistical Planning and Inference 15, 189-204.

Inderjit, J. C. \& Olofsdotter, M. (2002), 'Joint action of phenolic acid mixtures and its significance in allelopathy research', Physiologia Plantarum 114, 422-428.

Kiefer, J. (1959), 'Optimum experimental designs', Journal of the Royal Statistical Society 21, 272-319.

Kiefer, J. \& Wolfowitz, J. (1959), 'Optimum designs in regression problems', Annals of Mathematical Statistics 30(2), 271-294.

López, V. I. (2008), Diseños óptimos para discriminación y estimación en modelos no lineales, PhD thesis, Centro de Investigación en Matemáticas, A.C, Guanajuato, México.

López, V. I. \& Ramos, R. (2007), 'Una introducción a los diseños óptimos', Revista Colombiana de Estadística 30(1), 37-51.

O'Brien, T. (1992), 'A note on quadratic designs for nonlinear regression models', Biometrika 79, 847-859.

O'Brien, T. (1995), 'Optimal design and lack of fit in nonlinear regression models', Statistical modelling, Lecture Notes in Statistics 104, 201-206. 